\title{
Antimicrobial resistant in Gram-negative bacilli: Enterobacteriaceae and non-fermenting bacilli isolated at Sominé DOLO Hospital of Mopti, Mali
}

Moussa Diawara 1, *, Modibo Coulibaly 2, Dramane Samaké ${ }^{3}$, Soumaila Touré ${ }^{2}$, Dramane Cissé 4 , Abdoulaye Traoré ${ }^{5}$, Djibril Traoré ${ }^{4}$, Pierre Coulibaly ${ }^{6}$, Bréhima Traoré ${ }^{4}$, Oumar Guindo ${ }^{7}$, Bakary Maiga ${ }^{8}$ and Amagana Dolo ${ }^{9}$

${ }^{1}$ Department of Biomedical laboratory, Military hospital infirmary of Kati, Mali.

2 Department of Biomedical laboratory, Sominé DOLO Hospital of Mopti, Mali.

${ }^{3}$ Department of Medicine, Sominé DOLO Hospital of Mopti, Mali.

${ }^{4}$ Department of General surgery, Sominé DOLO Hospital of Mopti, Mali.

${ }^{5}$ Department of Anesthesia and resuscitation, Sominé DOLO Hospital of Mopti, Mali.

${ }^{6}$ Department of Gynecology and obstetrics, Sominé DOLO Hospital of Mopti, Mali.

7 Department of Public Health, Sominé DOLO Hospital of Mopti, Mali.

${ }^{8}$ Faculty of Medicine and Odonto-Stomatology, University of Technical and Technological Sciences, Mali.

${ }^{9}$ Faculty of Pharmacy, University of Technical and Technological Sciences, Mali.

GSC Biological and Pharmaceutical Sciences, 2022, 18(01), 008-013

Publication history: Received on 04 December 2021; revised on 04 January 2022; accepted on 06 January 2022

Article DOI: https://doi.org/10.30574/gscbps.2022.18.1.0021

\begin{abstract}
Background: $\beta$-lactams and carbapenems are the major antibiotics used to treat gram-negative bacteria and nonfermenting bacilli. However, the increasing production of $\beta$-lactamase and carbapenemase limits the therapeutic options. Our study aims to determine the resistant phenotypes of these bacteria while describing their epidemiological aspect.
\end{abstract}

Material and Methods: This was a cross-sectional study by consecutive enrollment from January 2018 to December 2019 at Sominé DOLO Hospital of Mopti, Mali. We performed manual method for bacteria culture, identification and antibiotics sensitivity testing. The antibiotics sensitivity testing was accessed by the diffusion method according to CASFM/EUCAST ("Comité de l'Antibiogramme de la Société Française de Microbiologie" / European Committee on Antimicrobial Susceptibility Testing) recommendations V1.0 february 2018 and V2.0 may 2019 2019 V.2.0. May recommendation released in 2019. Data were analyzed by software R 4.0.3 GUI 1.73 Catalina build (7892).

Results: At all 904 samples were included in this study. Out of the 904 cultures, 297 sample (32.85\%) were positive. The rates of enzymes production were as follow: Extended-spectrum ß-lactamase (ESBL) 56.42\% (101/179), cepholosporinase hyperproduction (HCASE) 15.64\% (28/179), cephalosporinase production (CASE) 6.14\% (11/179), penicillinase hyperproduction (HP) 5.58\% (10/179), carbapenemase production (CP) 6.14\% (11/179) and savage strains $10.05 \%(18 / 179)$.

Conclusion: Our data showed a high prevalence of resistance to $\beta$-lactamins and carbapenemes in gram-negative bacteria and non-fermenting bacilli. The A high level of $\beta$-lactamase and carbapenemase production by gram negative bacilli were also reported by others authors calls for the rational use of antibiotic in hospital setting.

Keywords: Gram-negative bacilli; Antimicrobial resistant; Rational use of antibiotics.

\footnotetext{
*Corresponding author: Dr. Moussa Diawara, Pharm.D; Email: moussadiaw13@gmail.com

Department of Biomedical laboratory, Military hospital infirmary of Kati, Mali. 


\section{Background}

Gram-negative bacilli, most notably Escherichia coli, Klebsiella pneumoniae, Pseudomonas aeruginosa, and Acinetobacter baumannii, are among the most important causes of serious hospital-acquired and community-onset bacterial infections in humans [1]. Resistant to antibiotics of these bacteria is increasing worldwide in healthcare settings and in the community [2]. Gram-negative bacteria because of their high resistant rate are among a global priority list of antibiotic-resistant bacteria to guide research, discovery and development of the new antibiotic [3]. The $\beta$-Lactam antibiotics are a major drug class used to treat serious community-onset or hospital-acquired infections caused by gram negative bacilli, resistance to these agents will continue to challenge clinical therapeutic choices [4]. Carbapenemase and $\beta$-lactamases are bacterial enzymes that inactivate $\beta$-lactam antibiotics by hydrolysis, which results in ineffective compounds. In Gram-negative bacilli, these enzymes production remains the most important contributing factor to $\beta$-lactam resistance and these dissemination is alarming [2]. Low and middle income countries (LMICs) are and will be particularly affected [5]. A few data concern antibiotic-drug-resistance is available in Mali, that is also a middle and income country and that not have a real antimicrobial resistance policy. The objective of this study was to determine the resistant phenotypes of gram negative bacteria while describing their epidemiological aspect at Sominé DOLO hospital of Mopti and the prevalence of these bacilli.

\section{Material and methods}

We enrolled a total of 904 samples for bacteriological analysis in a prospective study during 2 years January 2018 to December 2019 at Sominé DOLO hospital of Mopti (SDH-M)/Mali. SDH-M is the unique secondary health care setting in Mopti area, it's also in transition to third reference health care. In this study we included all bacteriological specimens sent to our laboratory for bacteria identification and antibiotics susceptibility (urine, stool, sperm, ascistes fluid, pleural fluid, prostatic fluid, throat swab, pus and others specimens). All patients with positive culture containing an Enterobacteriaceae or non-fermentative bacilli were therefore included in antibiotic susceptibility analysis. The manual method was used for bacteria culture, identification, and sensitivity testing. Uriselect ${ }^{\circledR}$ medium (a chromogen medium) served as a medium for culture and identification for bacteria isolated in urine combined with others biochemistry tests (oxydase, catalase, urease, indole) after microscopic observation to identified bacteria morphology. Bacteria isolated from others samples had grown on different mediums (Ordinary medium, Drigalski medium, Salmonella/Shigella mediums, and Hektoen medium). Analytical Profile Index 20-Enterobacteriaceae (API 20-E ${ }^{\circledR}$ ) and Analytical Profile Index 20- Non- Enterobacteriaceae (API-20 NE ${ }^{\circledR}$ ) systems (BioMérieux, Marcy l’Etoile, France) were used to identified bacteria isolated on specimens others that urines. Antibiotic susceptibility testing was performed by the diffusion method by agar-agar according to CA-SFM/ EUCAST ("Comité de l'Antibiogramme de la Société Française de Microbiologie" / European Committee on Antimicrobial Susceptibility Testing) recommendations V1.0 february 2018 and V2.0 may 20192019 V.2.0. May. Extended-spectrum $\beta$ lactamase (ESBL) production and Carbapenamase production by gram negative bacilli bacteria was detected by phenotypic method. This method is based on the principle that most ESBLs hydrolyze third generation of cephalosporins although they are inhibited by clavulanate. For ESBLs phenotypic detection, we used synergy method between two antibiotic discs on standard antibiogram, one disc of third or fourth generation of cephalosporin (cefotaxime, ceftriaxone, ceftazidime, cefepime) or aztreonam in combination with one disc of clavulanic acid at a distance of $30 \mathrm{~mm}$. ESBL presence is expressed by the appearance of a synergy $<<$ champagne cork $>>$. For a clinical isolated producers ESBL who had others $\beta$-lactam resistant mechanisms as cephalosporinase production, the synergy image was detected by the rapprochement of cephalosporin disc of the clavulanate acid disc or by performing a standard antibiogram on Mueller- Hinton agar supplemented with $250 \mathrm{mg} / \mathrm{L}$ of cloxacillin (cephalosporinase inhibitor). For carbapenemase detection we measured only carbapenem disc diameter. Data were captured in Excel Microsoft and analyzed by the statistical software R 4.0.3 GUI 1.73 Catalina build (7892).

\section{Results}

During the study period, we received 904 samples for bacteriological analysis. Out of the 904 cultures, 297 sample (32.85\%) were positive. At these positives cultures, $60.27 \%(179 / 297)$ were gram negative bacilli: Enterobacteria and non-fermentative bacilli, account for 53.20\% (158/297) and 7.07\% (21/297), respectively. Among the patients what we isolate gram negative bacilli, $63.13 \%$ (113/179) were female and $36.87 \%(66 / 179)$ were males. The mean age was 37 years with extremes to 1 to 90 years, and the age group of 31 to 45 years was predominant 27.93\% (Figure 1). Urine culture was the most performed analysis $82.68 \%(148 / 179)$, followed by pus culture 5,59\% (10/179) and sperm culture $5.03 \%$ (9/179) (Figure 2). Out of the 179 gram-negative bacilli, the most clinical bacteria isolates were Escherichia coli 61.45\% (110/179) , Klebsiella pneumonia 13.97\% (25/179) and Pseudomonas aeruginosa $7.26 \%$ (8/179) (Table 1). Antibiotic susceptibility test reveals that the main antibiotics resistance rates were as follow: 
amoxicillin 99.44\% (178/179), amoxicillin + clavulanic acid 98.88\% (177/179), third generation cephalosporine 79.89\% (143/179) (Figure 3). The rates of enzymes production were as follow: Extended-spectrum ß-lactamase (ESBL) $56.42 \%$ (101/179), cepholosporinase hyperproduction (HCASE) 15.64\% (28/179), cephalosporinase production (CASE) 6.14\% (11/179), penicillinase hyperproduction (HP) 5.58\% (10/179), carbapenemase production (CP) 6.14\% $(11 / 179)$ and savage strains $10.05 \%$ (18/179) Figure 4.

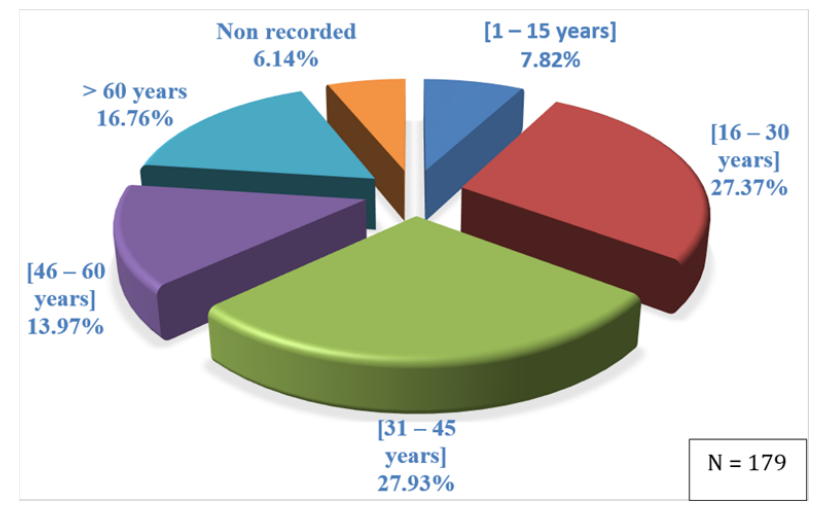

Figure 1 Age group proportions of study subjects

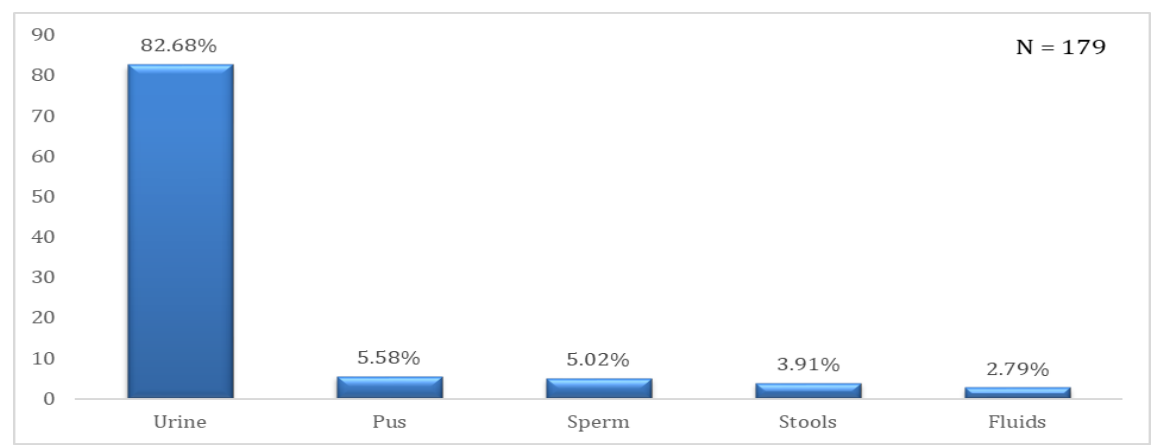

Figure 2 Proportions of received biological samples

Table 1 Distribution of clinical isolated bacteria

\begin{tabular}{|l|c|c|}
\hline \multicolumn{1}{|c|}{ Isolated spp } & N & \% \\
\hline Escherichia coli & 110 & 61.45 \\
\hline Klebsiella pneumoniae & 25 & 13.97 \\
\hline Pseudomonas aeruginosa & 13 & 7.26 \\
\hline Acinetobacter baumannii & 8 & 4.47 \\
\hline Enterobacter cloacae & 6 & 3.35 \\
\hline Salmonella spp & 5 & 2.79 \\
\hline Serratia marcescens & 3 & 1.68 \\
\hline Pseudomonas vulgaris & 1 & 0.56 \\
\hline Pseudomonas multocisa & 1 & 0.56 \\
\hline Pseudomonas luteola & 1 & 0.56 \\
\hline Pseudomonas damsele & 1 & 0.56 \\
\hline Citrobacter koresi & 1 & 0.56 \\
\hline Citrobacter braaki & 1 & 0.56 \\
\hline Total & 179 & 100 \\
\hline
\end{tabular}




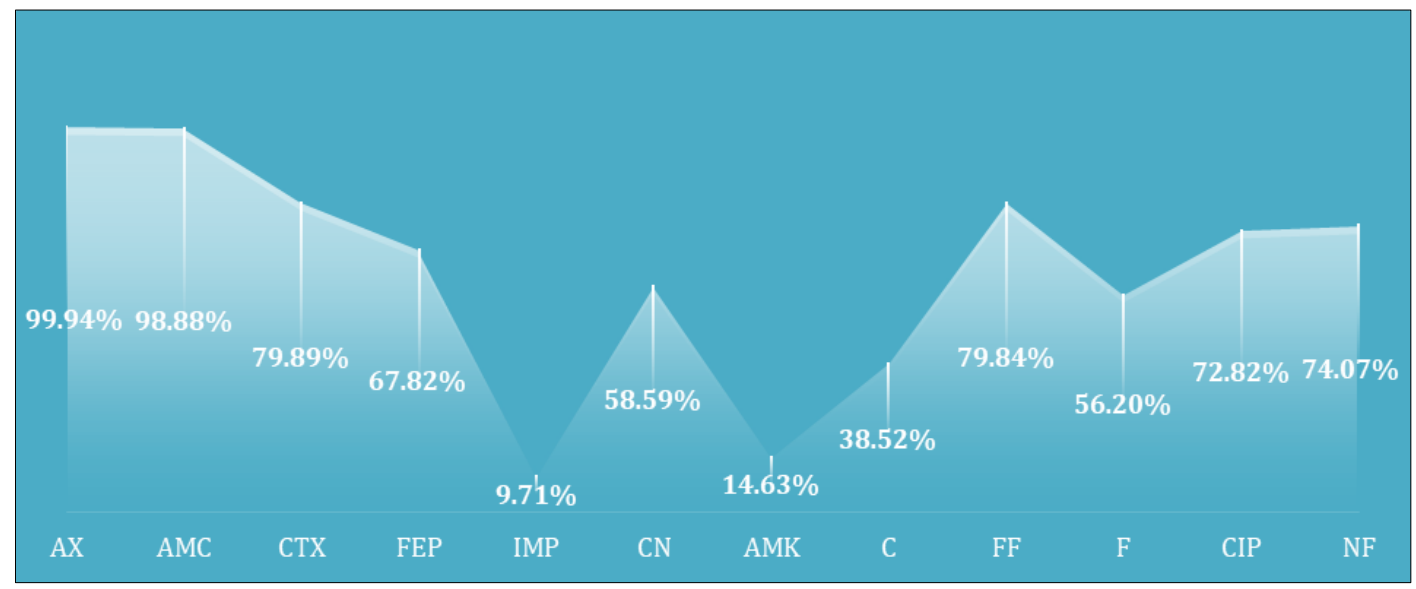

Figure $3 \mathrm{AX}=$ amoxicillin, $\mathrm{AMC}=$ amoxicillin + clavulanic acid, CTX = cefotaxime, $\mathrm{FEP}=$ cefepime, $\mathrm{IMP}=\mathrm{imipenem}, \mathrm{CN}$ = gentamycin, $\mathrm{AMK}=$ amikacin, $\mathrm{C}=$ chloramphenicol $\mathrm{FF}=$ Fosfomycin, $\mathrm{F}=$ nitrofurantoin, $\mathrm{CIP}=$ ciprofloxacin, $\mathrm{NF}=$ norfloxacin

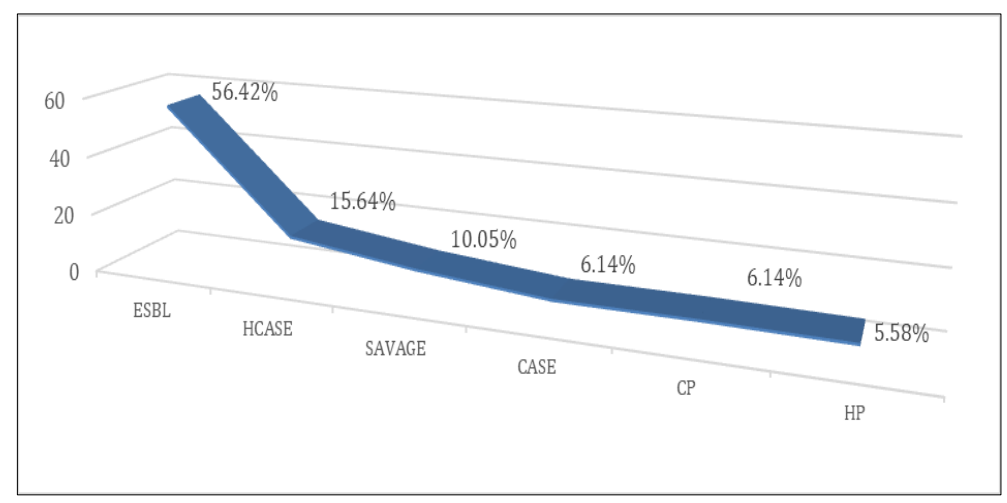

Figure 4 Distribution of resistant phenotypes. ESBL = extended-spectrum ß-lactamase, HCASE = high cephalosporinase, $\mathrm{CASE}=$ cephalosporinase, $\mathrm{CP}=$ carbapenemase, $\mathrm{HP}=$ penicillinase hyperproduction

\section{Discussion}

Globally the prevalence of urinary tract infection (UTI) caused by GNB was $12.16 \%$. This high UTI could probably due to the high proportion of female gender and to the low demand of others bacteriological samples addressed to the laboratory. In our study, the proportion of female was high $63.13 \%$ and the age group of 31 to 45 years was the most exposed. The gram negative bacilli (GNB) was isolated mainly in urinary culture $(82,68 \%)$, following by pus culture (5.59\%) and sperm culture (5.03\%). Escherichia coli and $61.54 \%$ and Klebsiella pneumoniae $13.97 \%$ were, respectively the most Enterobacteriaceae isolated in the urine culture. Pseudomonas aeruginosa and Acinetobacter baumanii respectively $7.26 \%$ and $4.47 \%$ were also the most non fermentative bacilli isolated also in the urine culture. Our data are in line with the that of Fissou Henry YANDAI and al (2019) [6] in Tchad where they found a rate of 18.5\% of urinary tract infection (UTI). Fissou Henry YANDAI and al identified E. coli and K. pneumoniae as the two major bacteria involved in UTI 64.5\% and 27.7\%, respectively. In Cameroun, Ebongue et al (2015) found the same result [7].

Our study reveals a high antibiotics resistance rate, globally $89.95 \%$. Strains with savage phenotype were isolated only in $10.05 \%$ of the culture samples. The analyses of the antibiotic susceptibility to $\beta$-lactam showed also a high resistance levels of Enterobacteriaceae and to non-fermentative bacilli : resistant to amoxicillin $99.44 \%$, resistant to amoxicillin + clavulanate acid $98.88 \%$, resistant to third generation cephalosporine $79.89 \%$, resistant to fourth generation cephalosporine $67.82 \%$ and resistant to imipenem $9.71 \%$. This result is in correlation with the study conducted by Samba Adama Sangare and al , 2017 in Mali where they found also a high antibiotics resistance rate to Enterobacteriaceae [8]. In our study, imipenem resistance to gram negative bacilli rate was high particularly $P$. aeruginosa $15,38 \%$, A. baumanii $12,50 \%$ and E. coli in $10.91 \%$. This high resistant level to imipenem could be due to the more and more increasing use of imipenem caused by the poor sensibility of hospital strains to third and fourth cephalosporine generation. The different phenotypes resistance rate to gram negative bacilli were as follow: ESBL 
(56.24\%), HCASE (15.64\%), CP (6.14\%), CASE (6.14\%), and HP (5.58\%). Among clinical isolates in Mopti hospital, the prevalence of ESBL producing gram negative bacilli (ESBL-PGNB) was very high (56.24\%). ESBL-PGNB prevalence varies widely between geographic areas. In Sub-Saharan Africa, and particularly in West Africa, limited data are available on ESBL-PGNB. The prevalence found in our study (56.74\%) was similar to the one reported for other African countries, such as Burkina Faso 58\% [9], Mali 61.8\% [10] and Ghana 49.4\% [11]. In others African countries, prevalence of ESBL-PGNB was relatively low such as Nigeria [12] and Central African Republic [13]. In our study, ESBL and CP affected antibiotics drug susceptibility such as aminosid and fluroquinolone. Samba Adama Sangare and al in Mali , 2017 [8], Oumar Ouchar Mahamat and al in Tchad [14] reported the same result. This study shed light on the different pathogenic bacteria isolated at Sominé DOLO hospital of Mopti and their antibiotic resistance phenotypes. However, we did not perform molecular testing to confirm the genotype of clinical isolates. The use of control strains would improve this job.

\section{Conclusion}

Escherichia coli 61,45\% (110/179) and Klebsiella pneumoniae 13,97\% (25/179) were the two majors clinical isolates isolated in clinical specimens particularly in urine. ESBL production by gram-negative bacilli was very high $56,42 \%$ and affect antibiotics which are not usually hydrolyzed by ESBLs such as carbapenem 6,14\% (11/179). This high level of carbapenemase reported by others authors calls for the rational use of antibiotic in hospital setting.

\section{Compliance with ethical standards}

\section{Acknowledgments}

We are grateful to all study subjects for participating in this study who gave their informed consent. We also thank all the staff of Mopti hospital for their frank and closed collaboration.

\section{Disclosure of conflict of interest}

Authors certify that there is no actual or potential conflict of interest in relation to this article.

\section{Statement of informed consent}

Each participant gave fully informed written consent prior to enrollment. The protocol was reviewed and approved by Hospital committee.

\section{References}

[1] Paterson DL. Resistance in gram-negative bacteria: Enterobacteriaceae. Am. J. Infect. Control. 2006; 34: S20-S28, S64-S73.

[2] Pitout JD, Laupland KB. Extended-spectrum beta-lactamase-producing Enterobacteriaceae: an emerging publichealth concern. Lancet Infect Dis. 2008; 8(3):159-66.

[3] World Health Organization 2017: global priority list of antibiotic resistant - bacteria to guide research, discovery, and development of news antibiotics. Accessed June 2, 2021: https://www.who.int/medicines/publications/WHO-PPL-Short_Summary_25Feb-ET_NM_WHO.pdf.

[4] Livermore DM, Woodford N. The beta-lactamase threat in Enterobacteriaceae, Pseudomonas and Acinetobacter. Trends Microbiol. 2006; 14: 413-420.

[5] The World Bank Annual Report 2016. Accessed November 12, 2021: https://openknowledge.worldbank.org/handle/10986/24985.

[6] Fissou Henry Yandai, Guelmbaye Ndoutamia, Bessimbaye Nadlaou and al. Prevalence and resistance profile of Escherichia coli and Klebsielle pneumoniae isolated from urinary tract infections in N'Djamena, Tchad. Int. J. Biol. Chem. Sci. 2019; 13(4): 2065-2073.

[7] Ebongue CO, Tsiazok MD, Nda Mefo'o JP, Ngaba GP, Beyiha G, Adiogo D. Evolution de la resistance aux antibiotiques des Entérobactéries isolées à l'hopital Général de Douala de 2005 à 2012. Pan. Afr. Med. J. $2015 ; 20$ : 227. 
[8] Sangare SA, Rondinaud E, Maataoui N, Maiga AI, Guindo I, Maiga A, et al. Very high prevalence of extendedspectrum beta-lactamase producing Enterobacteriaceae in bacteriemic patients hospitalized in teaching hospitals in Bamako,Mali. PLoS ONE . 2017; 12(2): e0172652.

[9] Ouedraogo AS, Sanou M, Kissou A, Sanou S, Solare H, Kabore F, et al. High prevalence of extended spectrum $\beta$ lactamase producing Enterobacteriaceae among clinical isolates in Burkina-Faso. BMC Infect Dis. 2016; 16 : 326.

[10] Samba Adama Sanagre, Almoustapha Issiaka Maiga, Ibrehima Guindo and al. Prevalence of ESBL-producing Enterobactericeae isolated from blood cultures in Mali. J Infect Dev Ctries. 2016; 10(10): 1059-1064.

[11] Obeng-Nkrumah N, Twum-Danso K, Krogfelt KA, Newman MJ. High levels of extended-spectrum beta-lactamases in a major teaching hospital in Ghana. The need to regular monitoring and evaluation of antibiotic resistance. AmJ Trop Med Hyg. 2013; 89(5): 960-4.

[12] Ogbolu DO, Daini AO, Ogunledun A, et al. High levels of multidrug resistance in clinical isolates of gram-negative pathogens from Nigeria. Int J Antimicrob Agents. 2011; 37(1): 62-6.

[13] Raymond B, Dahlia M, Alexandre M, et al. Increasing prevalence of antimicrobial resistance among Enterobacteriaceae uropathogens in Bangui. Central African Republic. J Infect Dev Ctries. 2009; 3(3): 187-90.

[14] Oumar Ouchar Mahamat, Monon Lounnas, Mallorie Hide al. High prevalence and characterization of extendedspectrum $\beta$-lactamse production Enterobacteriaceae in Chadian hospitals. BMC Infections Diseases. 2019; 19(1): 205. 